\title{
Pan-cancer analysis of tumor metabolic landscape associated with genomic alterations
}

\author{
Hongyoon Choi ${ }^{* *}$ and Kwon Joong $\mathrm{Na}^{2^{*}}$ (D)
}

\begin{abstract}
Although metabolic alterations are one of the hallmarks of cancer, there is a lack of understanding of how metabolic landscape is reconstituted according to cancer progression and which genetic alterations underlie its heterogeneity within cancer cells. Here, the configuration of the metabolic landscape according to genetic alteration is examined across 7648 subjects representing 29 cancers. The metabolic landscape and its reconfiguration according to the accumulated mutation maintained characteristics of their tissue of origin. However, there were some common patterns across cancers in terms of the association with cancer progression. Carbohydrate and pyrimidine metabolism showed the highest positive correlation with tumor metabolic burden and they were also common poor prognostic pathways in several cancer types. We additionally examined whether genetic alterations associated with the heterogeneity of metabolic landscape. Genetic alterations associated with each metabolic pathway differed between cancers, however, they were a part of cancer drivers in most cancer types.
\end{abstract}

Keywords: Tumor mutation burden, Metabolic landscape, Cancer metabolism, Pan-cancer analysis, Driver gene mutation

Cancer cells reorganize their metabolism to fulfill their biosynthetic requirements for tumor growth and proliferation [1]. Besides activation of aerobic glycolysis, other metabolic pathways including nucleotide, amino acid, and lipid metabolism are also activated in cancer cells to produce biosynthetic building blocks for malignant cellular proliferation although the degree of metabolic activation is diverse $[2,3]$. Although recent studies have elucidated the metabolic reprogramming of cancer cells compared with normal tissues and its biological implications [2, 4], the configuration of metabolic landscape according to cancer progression in terms of tumor mutational burden (TMB) and clinical outcome is yet unclear. Moreover, the diversity of cancer cell metabolism is hardly explained by the conventional analogy of genetic alteration model that tumor metabolism simply supports tumor growth and proliferation [5, 6]. A comprehensive understanding of

\footnotetext{
* Correspondence: chy1000@snu.ac.kr; kjna85@gmail.com

${ }^{1}$ Department of Nuclear Medicine, Seoul National University Hospital, 101 Daehak-ro, Jongno-gu, Seoul 03080, Republic of Korea

2Department of Thoracic and Cardiovascular Surgery, Seoul National University Hospital, 101 Daehak-ro, Jongno-gu, Seoul 03080, Republic of Korea
}

genetic alterations which underlie the heterogeneity of the metabolic landscape of cancer cells can elucidate therapeutic targets in terms of cancer metabolism.

Here, we aim to investigate the metabolic landscape of multiple cancer types and its configuration according to the progression. We also aim to find answers for whether there are common features in genetic alterations related to the metabolic landscape across cancer types. An integrated analysis of genomic and transcriptomic profiles of 29 different solid cancers was performed. We demonstrate that some common metabolic pathways exhibit the close relationship with TMB and clinical outcome across several cancer types. We also show that genetic alterations associated with cancer metabolic heterogeneity were a part of cancer drivers in most cancer types.

\section{Results and Discussions \\ Metabolic landscape specific for cancer types}

We compared the median values of metabolic signatures manually curated considering cancer-related pathways for each cancer type (Additional file 1: Figure S1A). Metabolic profiles of all cancers were visualized by t-SNE [7] 


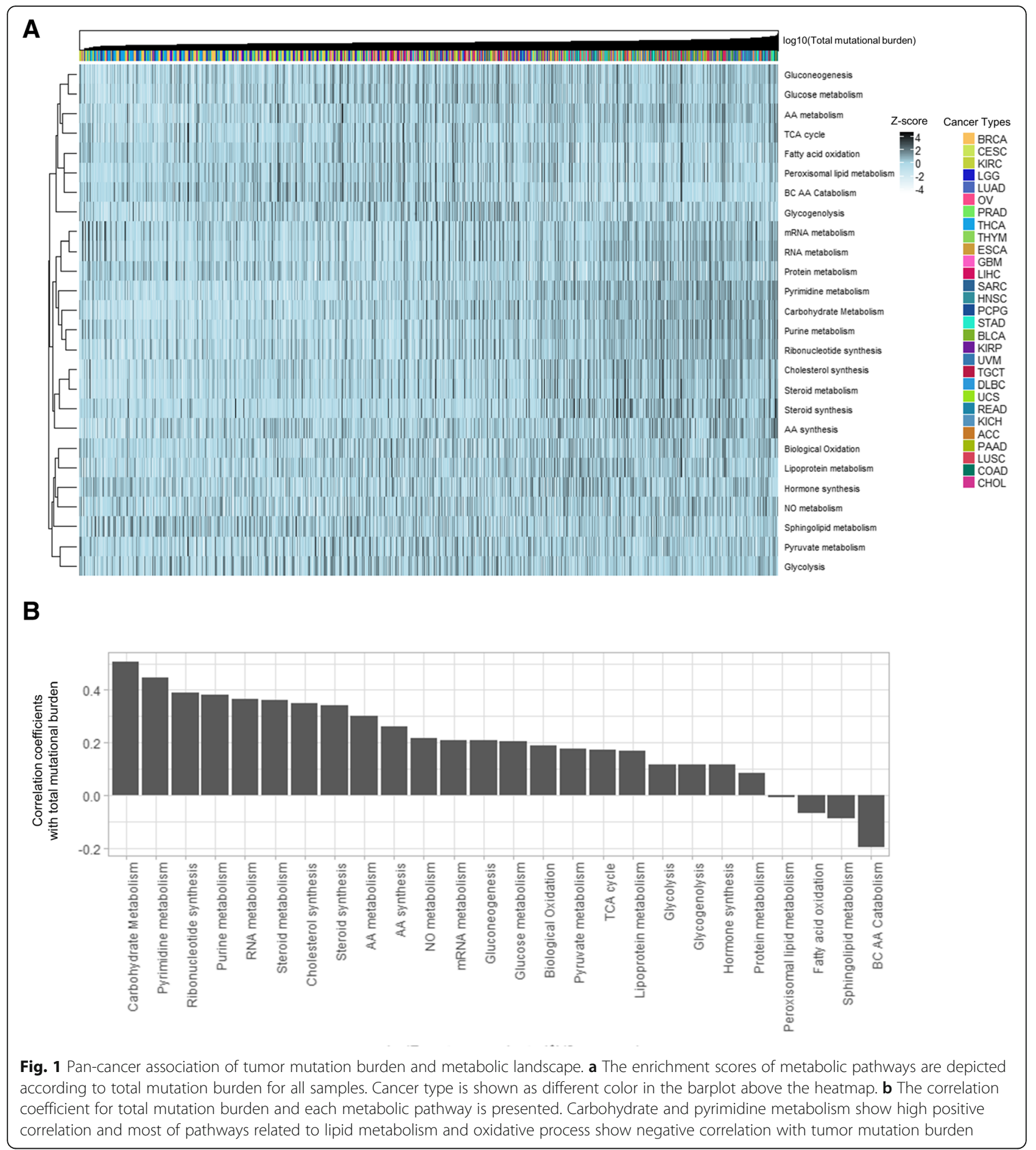

(Additional file 1: Figure S1B). Metabolic profiles of cancer tissues were clustered according to each cancer type. Additionally, metabolic profiles were closely mapped with those of same histologic types (e.g. HNSC, LUSC, and CESC clusters.) Of note, metabolic profiles of TCGT and CHOL showed heterogeneous metabolic characteristics. TGCT showed two large clusters, which correspond to histologic subtypes, and $\mathrm{CHOL}$ showed more heterogeneous metabolic profiles than others (Additional file 1: Figure S2).

Metabolic reconstitution according to mutational burden Metabolic signatures of all samples were ordered by TMB and presented by a heatmap (Fig. 1a). Carbohydrate metabolism showed the highest correlation with TMB 
A

Pyrimidine metabolism Carbohydrate Metabolism Glycolysis

Purine metabolism

RNA metabolism

Lipoprotein metabolism

Pyruvate metabolism

Ribonucleotide synthes

No metabolism

Hormone synthesis

Glucose metabolism

Cholesterol synthesis

Biological Oxidation

AA metabolism

Glycogenolys

TCA cycle
Steroid metabolism

Gluconeogenesis

AA synthesis

Protein metabolism

Sphingolipid metabolism

Peroxisomal lipid metabolism

BC AA Catabolism
Fatty acid oxidation

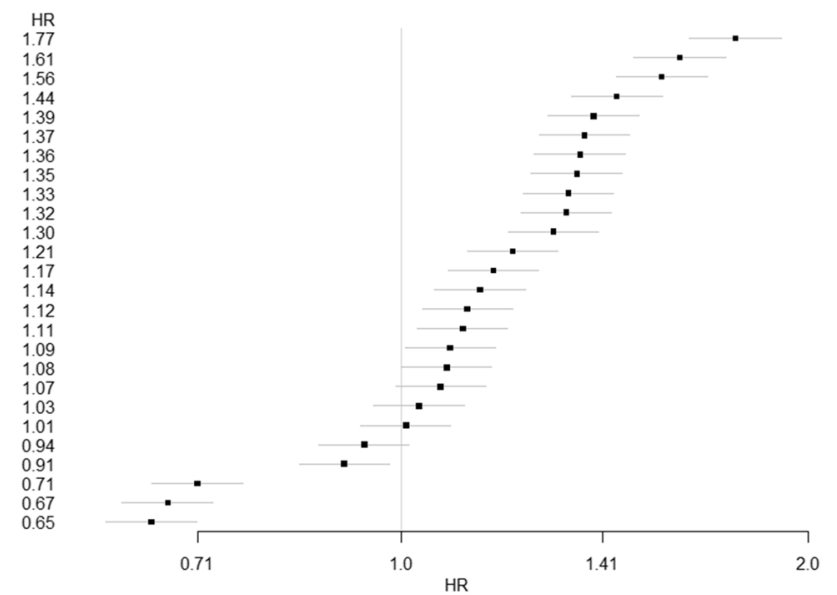

B

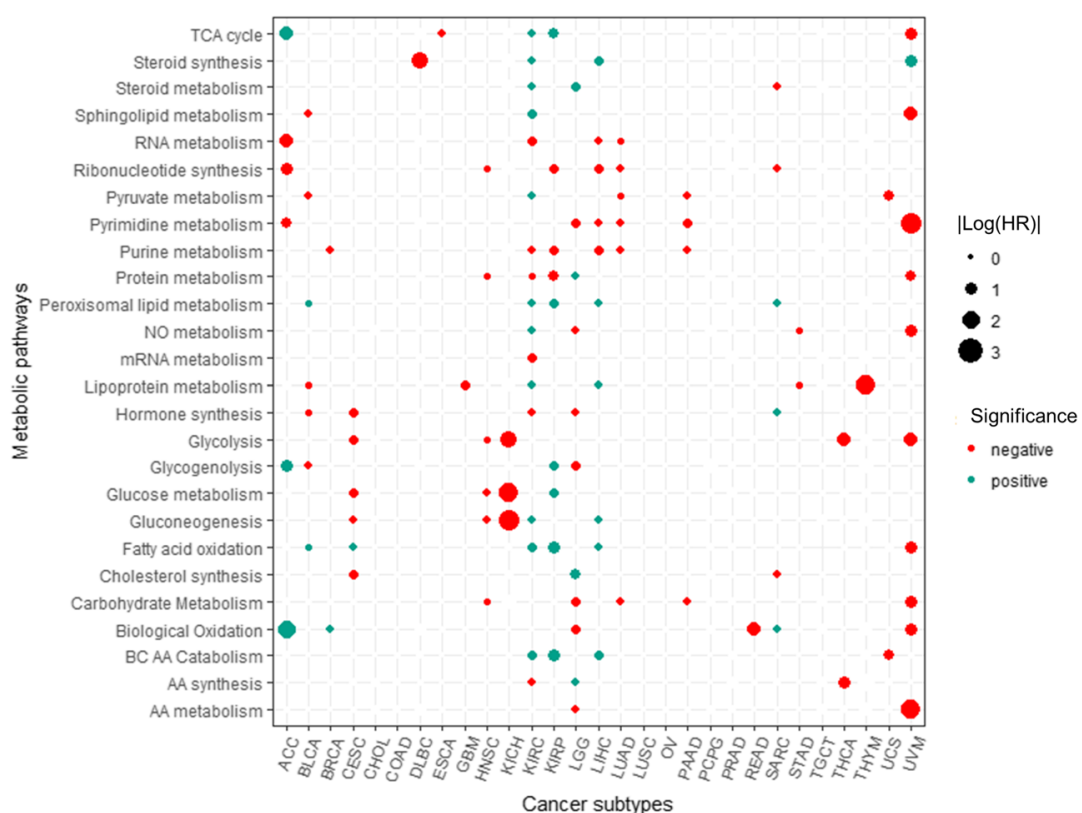

C

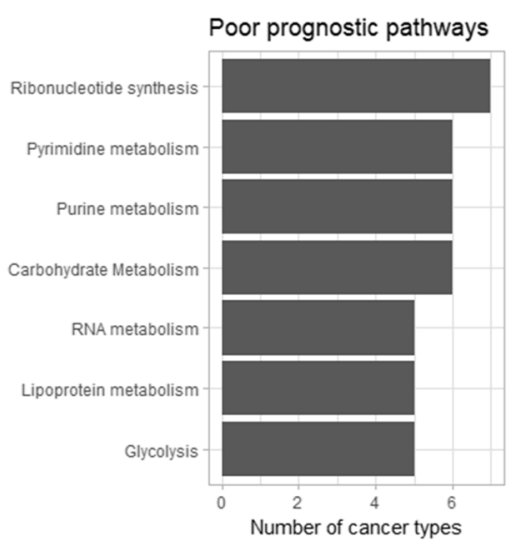

D

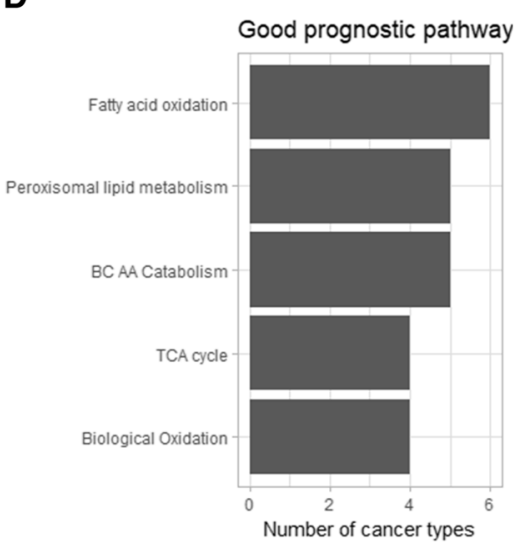

Fig. 2 (See legend on next page.) 
(See figure on previous page.)

Fig. 2 Prognostic significance of metabolic pathways. a Hazard ratios from pan-cancer analysis of each metabolic pathways to overall survival are shown. $\mathbf{b}$ The bubble plot shows the result of Cox proportional analysis for each cancer type. Only significant $(p<0.05)$ metabolic pathways are shown in this plot. The size of circle represents the log-scaled hazard ratio, and the color of circle represents negative (red) or positive (blue) prognostic significance. $\mathbf{c}$, d Frequency of metabolic pathways found negative (c) or positive (d) prognostic significance

followed by pyrimidine metabolism (Fig. $1 \mathrm{~b} ; p<0.05$ for all pathways). Of note, glycolysis, the well-known cancer metabolic feature, showed relatively weak correlation with TMB. LUSC showed the highest carbohydrate metabolism and TMB among 29 cancer types (Additional file 1: Figure S3). We could find a trend of increased carbohydrate and pyrimidine metabolism according to increased TMB across cancer types (Additional file 1: Figure S4). Of note, carbohydrate metabolism pathways included broad metabolic pathways related to energy metabolism including glycogen, glucose, disaccharides, pentose phosphate, and glycosaminoglycan, thus, the association with TMB suggested comprehensive changes in various energy metabolism pathways. We also investigated whether cancer types differ in the association between the metabolic landscape and TMB. Most cancer types showed a positive correlation between TMB and carbohydrate metabolism, while THYM and UVM showed a negative correlation (Additional file 1: Figure S5). Notably, TMB of these two cancer types was relatively lower than other cancer types.
Recently, TMB has been regarded as an important predictive biomarker for cancer immunotherapy, based on the idea that highly mutated tumors harboring more neoantigens can be targets of activated immune cells [8, 9]. In this regard, metabolic reconfiguration according to TMB can be an additional biomarker for cancer immunotherapy as tumor metabolism can be noninvasively and macroscopically estimated by PET. Moreover, the important metabolic signatures of each cancer type could help us find appropriate surrogate markers for PET radiotracers.

\section{Metabolic landscape reveals prognostic pathways}

Several metabolic signatures were associated with overall survival across all cancer samples (Fig. 2a; $p<0.05$ for all pathways). In general, carbohydrate and nucleotide metabolism were associated with poor prognosis. Specifically, poor prognostic metabolic signatures include carbohydrate metabolism, ribonucleotide synthesis, pyrimidine metabolism, purine metabolism, and glycolysis, whereas good prognostic signatures include peroxisomal

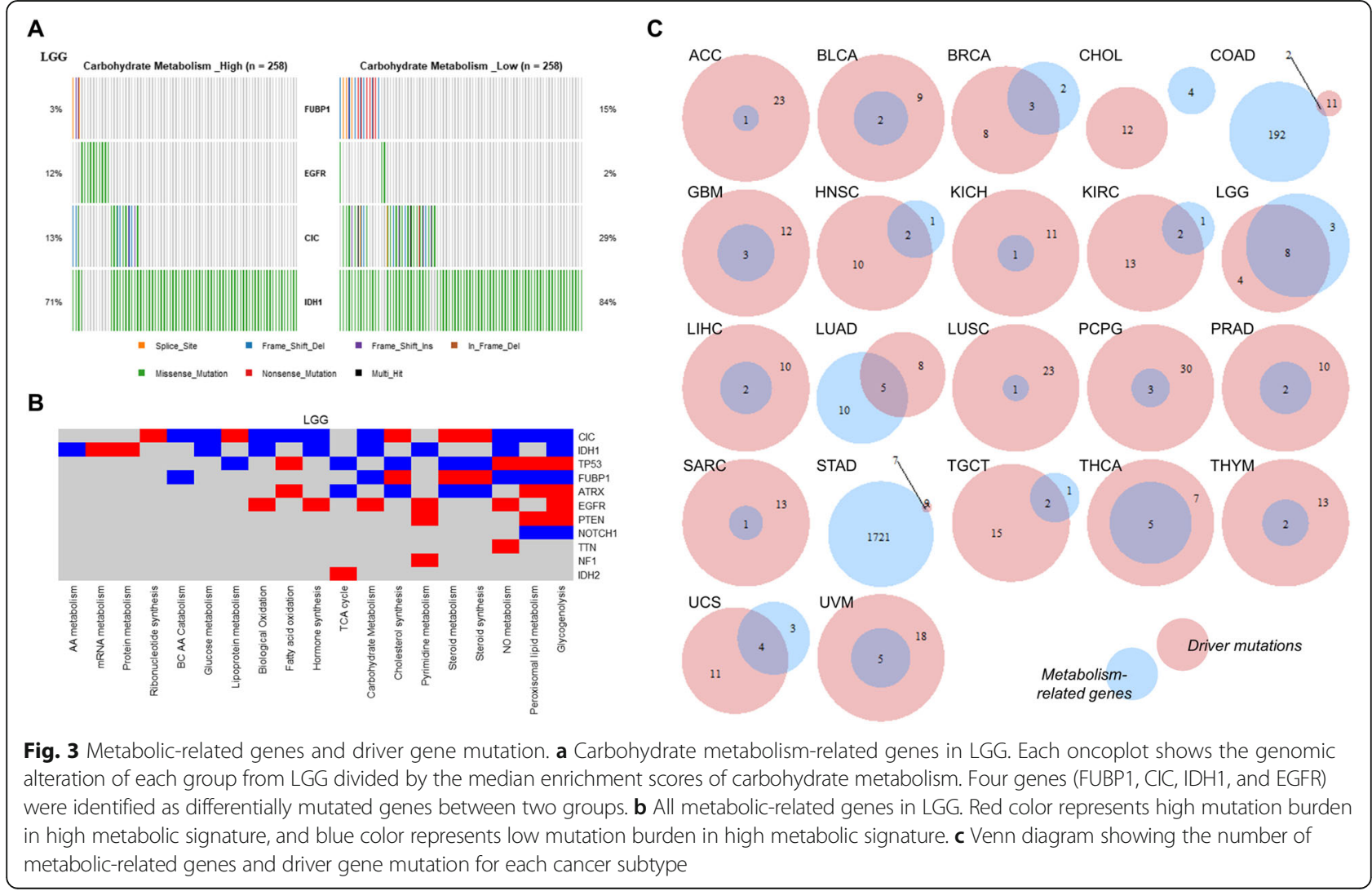


lipid metabolism, fatty acid oxidation, and biological oxidation.

Metabolic signatures significantly associated with prognosis for each cancer type were presented in Fig. 2b. The most common metabolic signatures significantly associated with poor prognosis were ribonucleotide synthesis (7 of 29 cancers), followed by pyrimidine, purine, and carbohydrate metabolism (6 or 29 cancers) (Fig. 2c). The metabolic signatures significantly associated with good prognosis were fatty acid oxidation ( 6 of 29 cancers), peroxisomal lipid metabolism, and branched chain amino acids catabolism (5 of 29 cancers) (Fig. 2d).

In brief, the increased metabolic activity of carbohydrate and nucleotides in cancer was associated with poor prognosis as well as the progression of cancer in terms of TMB as found in previous results. Furthermore, our results were similar with the previous report, which showed that glycolysis, ribonucleotide, pyrimidine, and purine metabolism were associated with poor prognosis, and mitochondrial fatty acid oxidation and peroxisomal lipid metabolism were associated with good prognosis [2]. These results support the idea that the link between biological stepwise mutational progression, metabolic landscape reconfiguration, and clinical progression of cancer. Accordingly, aggressive malignant phenotypes of cancer cells obtained by accumulated mutations change metabolic phenotypes to demand energy source represented by carbohydrate and elements for proliferation represented by nucleotide.

\section{Metabolism-related genetic alterations are mostly cancer drivers}

As cancer cells exhibit various metabolic landscapes even in same cancer types, genetic alterations could underlie this variety. We tried to examine the genetic alterations related to the metabolic landscape of each cancer type to investigate whether there is a common feature in the metabolism-related genetic alterations across several cancer types. An example of carbohydrate metabolism-related genetic alterations of LGG is presented (Fig. 3a.) The tumors with high carbohydrate metabolism showed significantly more mutations of EGFR and fewer mutations of FUBP1, CIC, and IDH1. The genetic alterations of LGG related to other metabolic signatures are summarized in Fig. 3b. We defined differentially-mutated genes of tumors for at least one metabolic signature as the metabolism-related genes. All metabolism-related genes of each cancer type are presented in Additional file 1: Figure S6. Seven cancer types (CESC, DLBC, ESCA, KIRP, OV, PAAD, and READ) showed no metabolism-related gene.

We further examined whether the metabolism-related genes were cancer driver genes. All metabolism-related genes of 12 cancer types (ACC, BLCA, GBM, KICH,
LIHC, LUSC, PCPG, PRAD, SARC, THCA, THYM, and UVM) were included in cancer drivers (Fig. 3c). In other cancer types, most of the metabolism-related genes overlapped with cancer drivers except STAD, COAD, and CHOL. The metabolism-related genes of $\mathrm{CHOL}$ did not overlap with cancer drivers. Notably, metabolic profiles of $\mathrm{CHOL}$ were highly heterogeneous (Additional file 1: Figure S2) as a previous result. STAD and COAD showed a large number of the metabolism-related genes than other cancer types. Since they are known to include tumors with MSI which is associated with hypermutation $[10,11]$, we examined the relationship between metabolic profiles of the two cancer types and MSI status. As shown in Additional file 1: Figure S7, tumors with high metabolic signatures including pyrimidine metabolism and glucose metabolism were clustered in those with MSI.

According to the results, cancer transcripts and protein networks consisting of metabolic pathways may be changed by cancer drivers instead of alterations of genes participated in the networks. It corresponds to the Darwinian selection of cancer cells which carry driver mutations facilitate cellular survival and growth by changing a favorable metabolic landscape [12]. The results also suggest that the metabolic change as a hallmark of cancer may be one of the tumorigenesis processes caused by genetic alterations rather than an independent process.

\section{Conclusions}

Among several metabolic signatures, carbohydrate metabolism representing overall nutrient demand and nucleotide metabolism showed the closest association with TMB and clinical outcome. Genetic alterations closely associated with metabolic heterogeneity were a part of cancer drivers in most cancers rather than genes affiliated to metabolic pathways. It supports the role of cancer drivers in the evolution process in constituting metabolic landscape appropriate for tumor growth. Our database of the metabolic landscape (https://choih.shinyapps.io/metabolicsignatures) according to cancer type and its reconstitution according to cancer progression can contribute to developing appropriate diagnostics and therapeutics targeting metabolism for cancer subtypes.

\section{Additional file}

Additional file 1: Materials and methods, supplementary figures and supplementary tables. (PDF $1650 \mathrm{~kb}$ )

Abbreviations

MSI: Microsatellite instability; TCGA: The Cancer Genome Atlas; TMB: Tumor mutation burden; t-SNE: T-Distributed Stochastic Neighbor Embedding 


\section{Abbreviations for cancer type}

ACC: Adrenocortical carcinoma; BLCA: Bladder urothelial carcinoma; CESC: Cervical and endocervical cancers; CHOL: Cholangiocarcinoma; COAD: Colon adenocarcinoma; DLBC: Diffuse Large B-cell Lymphoma; ESCA: Esophageal carcinoma; GBM: Glioblastoma multiforme; HNSC: Head and Neck Squamous Cell Carcinoma; KICH: Kidney Chromophobe; KIRP: Kidney renal papillary cell carcinoma; LGG: Brain low grade glioma; LIHC: Liver hepatocellular carcinoma; LUSC: Lung Squamous Cell Carcinoma; OV: Ovarian serous cystadenocarcinoma; PAAD: Pancreatic adenocarcinoma; PCPG: Pheochromocytoma and Paraganglioma; PRAD: Prostate adenocarcinoma; READ: Rectum adenocarcinoma; SARC: Sarcoma; STAD: Stomach adenocarcinoma; TCGT: Testicular Germ Cell Tumors; THCA: Thyroid carcinoma; THYM: Thymoma; UVM: Uveal Melanoma

\section{Abbreviations for genes}

CIC: Capicua Transcriptional Repressor; EGFR: Epidermal growth factor receptor; FUBP1: Far Upstream Element Binding Protein 1; IDH1: Isocitrate dehydrogenase 1

\section{Acknowledgements}

The results here are in whole based upon data generated by the TCGA Research Network: "http://cancergenome.nih.gov/".

\section{Funding}

This research received no specific grant from any funding agency in the public, commercial, or not-for-profit sectors.

\section{Availability of data and materials}

The raw sequencing and clinical data can be found at the GDC portal (https://portal.gdc.cancer.gov/). The mutation data can be downloaded from the R library, 'TCGAmutations' (https://github.com/PoisonAlien/

TCGAmutations). Our processed results are available at a web-based resource (https://choih.shinyapps.io/metabolicsignatures). Software and resources used for the analyses are described in each method section.

\section{Authors' contributions}

$\mathrm{CH}$ and NKJ designed the study, analyzed and interpreted the data, wrote and edited manuscript, and read and approved the manuscript.

\section{Ethics approval and consent to participate}

Patient data we used were acquired by a publicly available dataset that removed patient identifiers. The publicly available data were collected with patients' informed consent approved by the institutional review boards of all participating institutions following 1964 Helsinki declaration and its later amendments or comparable ethical standards.

\section{Consent for publication}

Not applicable.

\section{Competing interests}

The authors declare that they have no competing interests.

\section{Publisher's Note}

Springer Nature remains neutral with regard to jurisdictional claims in published maps and institutional affiliations.

Received: 17 July 2018 Accepted: 25 September 2018

Published online: 17 October 2018

\section{References}

1. Hanahan D, Weinberg RA. Hallmarks of cancer: the next generation. Cell. 2011;144:646-74

2. Gaude E, Frezza C. Tissue-specific and convergent metabolic transformation of cancer correlates with metastatic potential and patient survival. Nat Commun. 2016;7:13041.

3. Boroughs LK, DeBerardinis RJ. Metabolic pathways promoting cancer cell survival and growth. Nat Cell Biol. 2015;17:351.

4. Haider S, Mclntyre A, van Stiphout RG, Winchester LM, Wigfield S, Harris AL, Buffa FM. Genomic alterations underlie a pan-cancer metabolic shift associated with tumour hypoxia. Genome Biol. 2016;17:140.
5. Cantor JR, Sabatini DM. Cancer cell metabolism: one hallmark, many faces. Cancer Discov. 2012;2:881-98.

6. Hsu PP, Sabatini DM. Cancer cell metabolism: Warburg and beyond. Cell. 2008:134:703-7.

7. Lvd M, Hinton G. Visualizing data using t-SNE. J Mach Learn Res. 2008;9: 2579-605.

8. Gibney GT, Weiner LM, Atkins MB. Predictive biomarkers for checkpoint inhibitor-based immunotherapy. Lancet Oncol. 2016;17:e542-51.

9. Rizvi NA, Hellmann MD, Snyder A, Kvistborg P, Makarov V, Havel JJ, Lee W, Yuan J, Wong P, Ho TS. Mutational landscape determines sensitivity to PD-1 blockade in non-small cell lung cancer. Science. 2015;348:124-8.

10. Cancer Genome Atlas Network. Comprehensive molecular characterization of gastric adenocarcinoma. Nature. 2014;513:202.

11. Cancer Genome Atlas Network. Comprehensive molecular characterization of human colon and rectal cancer. Nature. 2012:487:330.

12. Vogelstein B, Papadopoulos N, Velculescu VE, Zhou S, Diaz LA, Kinzler KW. Cancer genome landscapes. Science. 2013;339:1546-58.

\section{Ready to submit your research? Choose BMC and benefit from:}

- fast, convenient online submission

- thorough peer review by experienced researchers in your field

- rapid publication on acceptance

- support for research data, including large and complex data types

- gold Open Access which fosters wider collaboration and increased citations

- maximum visibility for your research: over $100 \mathrm{M}$ website views per year

At $\mathrm{BMC}$, research is always in progress.

Learn more biomedcentral.com/submissions 